\title{
BMJ Open A randomised trial of non-mydriatic ultra-wide field retinal imaging versus usual care to screen for diabetic eye disease: rationale and protocol for the Clearsight trial
}

Selina L Liu, ${ }^{1,2}$ Lewis W Mahon, ${ }^{2}$ Neil S Klar, ${ }^{3}$ David C Schulz, ${ }^{4}$ John R Gonder, ${ }^{4,5}$ Irene M Hramiak, ${ }^{1,2}$ Jeffrey L Mahon ${ }^{1,2,3}$

To cite: Liu SL, Mahon LW, Klar NS, et al. A randomised trial of non-mydriatic ultrawide field retinal imaging versus usual care to screen for diabetic eye disease: rationale and protocol for the Clearsight trial. BMJ Open 2017;7:e015382. doi:10.1136/ bmjopen-2016-015382

- Prepublication history and additional material are available. To view these files please visit the journal online (http://dx.doi. org/10.1136/bmjopen-2016015382).

Received 1 December 2016 Revised 30 May 2017

Accepted 10 July 2017

\section{CrossMark}

${ }^{1}$ Division of Endocrinology \& Metabolism, Department of Medicine, Schulich School of Medicine and Dentistry, Western University, London, ON, Canada ${ }^{2}$ St. Joseph's Health Care London, London, ON, Canada ${ }^{3}$ Department of Epidemiology and Biostatistics, Schulich School of Medicine and Dentistry, Western University, London, ON, Canada

${ }^{4}$ Department of Ophthalmology, Schulich School of Medicine and Dentistry, Western University, London, ON, Canada

${ }^{5}$ Ivey Eye Institute, St. Joseph's Health Care London, London, ON, Canada

Correspondence to

Dr Selina L Liu;

selina.liu@sjhc.london.on.ca

\section{ABSTRACT}

Introduction Suboptimal screening for diabetic eye disease is a major cause of preventable vision loss. Screening barriers include mydriasis and the extra time patients need to attend dedicated eye screening appointments. In the Clearsight trial, we are testing whether screening by non-mydriatic ultra-wide field (NM UWF) imaging on the day patients attend their diabetes outpatient clinic visit improves detection of clinically important eye disease compared with usual screening. Methods and analysis Patients with diabetes due for a screening eye exam by the 2013 Canadian Diabetes Association (CDA) practice guidelines are being randomised to on-site screening by NM UWF imaging on the day of their clinic visit or to usual screening where, per CDA guidelines, they are encouraged to arrange an exam by an optometrist. The primary outcome is actionable eye disease (AED) based on a need for referral to ophthalmology and/or increased ocular surveillance. The primary analysis will use an intention-to-screen approach that compares the proportions of detected AED between on-site and usual screening groups under a superiority hypothesis in favour of on-site screening. With 740 randomised participants, the study will have $80 \%$ power to detect $\geq 5 \%$ absolute increase in the AED rate among on-site screening versus usual screening participants. This difference translates into a number-needed-to-screen by on-site screening of 20 to detect 1 additional person with AED.

Ethics and dissemination The protocol was approved by the institutional review board of Western University. The findings of the trial will be disseminated directly to participants and through peer-reviewed publications and conference presentations.

Trial registration number ClinicalTrials.Gov NCT02579837 (registered 16 0ctober 2015).

Protocol issue date 18 November 2015.

\section{INTRODUCTION}

Diabetic retinopathy (DR) and diabetic macular oedema (DME) are major causes of vision loss. ${ }^{12}$ Effective treatments include better glycaemic and blood pressure

\section{Strengths and limitations of this study}

- The Clearsight trial is a pragmatic randomised study designed to assess the effectiveness of on-site non-mydriatic ultra-wide field (NM UWF) imaging in routine clinical practice.

- Comparing rates of detection of clinically important diabetic eye disease between patients randomised to NM UWF imaging and current screening strategies provides the most valid assessment of the relative advantages of NM UWF.

- The trial is not designed to test whether NM UWF imaging improves visual outcomes.

- The trial is being undertaken in patients who are being followed in a diabetes referral outpatient clinic and may not therefore be generalisable to primary care settings.

control, laser photocoagulation, intraocular vascular endothelial growth factor (VEGF) inhibitors and fenofibrate. ${ }^{3}$ Patients with DR and DME are often asymptomatic, which makes eye screening important. ${ }^{3}$ However, while major guideline groups including the National Institute for Health and Care Excellence (NICE), American Diabetes Association (ADA) and Canadian Diabetes Association (CDA) endorse screening, ${ }^{3-5}$ there is less agreement on how to screen. For example, NICE recommends dilated pupil digital retinal photography by trained staff ${ }^{4}$; ADA recommends a dilated pupil exam by an eye care professional or, as a second choice, retinal photography ${ }^{5}$; and CDA recommends screening by experienced professionals by any one of dilated pupil direct ophthalmoscopy or indirect slit-lamp funduscopy, digital fundus photography or 7-field stereoscopic colour fundus photography. ${ }^{3}$ 
The expected benefit of diabetic eye screening has not circumvented generally poor screening adherence rates. $^{6-8}$ Ocular screening technologies have evolved that address specific patient barriers including the need for mydriasis and the extra time to attend eye exams over other diabetes appointments. ${ }^{9-15}$ In these ways, non-mydriatic ultra-wide field (NM UWF) digital retinal imaging is attractive. ${ }^{16-20}$ It yields retinal views of up to 200 internal degrees $(\sim 80 \%$ of the retina) without mydriasis in a single image, can be done quickly by on-site technicians but read later by off-site eye care professionals and could therefore be an acceptable add-on test for patients who are found, at the time of their usual diabetes clinic visit, to be due for screening. Moreover, four well-designed observational studies have compared NM UWF imaging to other currently used diabetic eye screening tests. ${ }^{16-19}$ These studies-which are described in detail in online supplementary appendix 1 -show that NM UWF imaging's capacity to detect diabetic eye disease versus other procedures including the gold standard of 7-field stereoscopic fundus photography is generally very good based on levels of agreement by the kappa statistic, sensitivities, specificities and positive and negative predictive values. ${ }^{16-19}$ In comparison to current eye screening tests, NM UWF imaging therefore appears to mitigate patient barriers to screening without unacceptable losses in discriminative accuracy.

Despite its promise, screening for diabetic eye disease by NM UWF imaging has not been widely endorsed. This may reflect lack of definitive evidence showing that eye screening in routine clinical practice by NM UWF imaging is superior to current screening strategies. We have therefore designed and initiated a pragmatic randomised trial of NM UWF imaging versus usual screening for diabetic eye disease called Clearsight. We describe here the Clearsight trial protocol and also the results of a pilot study that we completed to guide development of the protocol.

\section{METHODS AND ANALYSIS \\ Clearsight trial protocol \\ Overview}

Clearsight is a single centre, randomised, parallel group, non-masked, controlled, superiority trial being conducted at a diabetes referral outpatient clinic in London, Ontario. Our hypothesis is that offering NM UWF imaging to patients who are due for eye screening when they attend their usual diabetes care visit will improve detection of clinically important eye disease compared with current practice. We are randomising diabetes patients who are due for an eye exam to on-site screening by the Optos 200Tx UWF retinal camera on the day of their clinic visit or to usual screening as that is recommended by CDA. The first participant was randomised in March 2016. The projected completion date is December 2019.

\section{Objectives}

Our primary objective is to determine whether patients undergoing on-site screening have a higher rate of detection of diabetic eye disease (DR and DME) needing referral to an ophthalmologist and/or more frequent surveillance ('actionable eye disease' (AED)) compared with participants undergoing usual screening. Because we are unsure about NM UWF imaging's capacity to reliably detect DME (vide infra), as secondary objectives we will assess whether: (a) addition of optical coherence tomography (OCT) improves detection of DME over NM UWF imaging alone and (b) OCT and NM UWF imaging improve detection of AED over usual screening. As an additional study objective, we will obtain data needed for an economic evaluation that will assess the incremental cost-effectiveness of on-site screening with NM UWF imaging versus usual screening for diabetic eye disease.

DME includes clinically significant macular oedema (CSME) by visual assessment of retinal thickening and presence of peri-foveal hard exudates and centre-involving DME based on measurement of retinal thickness at the macula and fovea by OCT. ${ }^{21} 22$ It is an important and treatable cause of vision loss. In the studies summarised in the online appendix comparing NM UWF imaging to other tests, while NM UWF imaging detected both DR and DME, its capacity to detect the latter may not have been sufficient to support use of NM UWF imaging by itself. ${ }^{16-19}$ For example, in the comprehensive study by Silva et al, the sensitivity of NM UWF imaging for CSME compared with 7-field fundus photographs was $76 \%,{ }^{19}$ which would mean that over $20 \%$ of patients screened solely by NM UWF imaging may have undetected but potentially treatable CSME. OCT more accurately detects DME, but we found no studies comparing NM UWF to OCT.

\section{Eligibility}

Patients with documented type 1 diabetes (T1D) or type 2 diabetes (T2D) who are seen in the outpatient diabetes clinic are potentially eligible for the trial. Entry criteria generally follow the 2013 CDA clinical practice guidelines for screening for diabetic eye disease in adults with diabetes where the recommendations are: (a) initial screening in all patients with newly diagnosed T2D and in patients over age 15 years with $\mathrm{T} 1 \mathrm{D} \geq 5$ years duration, (b) rescreening every year in patients with T1D who do not have DR and (c) rescreening every 1-2 years in patients with T2D who do not have DR. ${ }^{3}$

The inclusion criteria are: (a) provision of informed consent, (b) age $\geq 18$ years, (c) a diagnosis of T1D for $\geq 5$ years or T2D of any duration and (d) at least 12 months since the last screening for diabetic eye disease by an eye care professional. The exclusion criteria are: (a) under active follow-up by an ophthalmologist where this means that, at the time of recruitment, the patient is scheduled for a future ophthalmologist appointment for any reason; (b) inability to provide informed consent; and (c) any condition or circumstance that makes it unlikely in the 
investigator's judgement that the patient can adhere to the study protocol.

\section{Assignment of interventions}

We are using a computer-generated randomisation schedule to assign participants in a 1:1 ratio to on-site screening or usual screening. We are stratifying participants by glycaemic control (A1C $\leq 7.0 \%$ vs $>7.0 \%$ ) and previous treatment (any one of laser therapy, vitrectomy, intraocular anti-VEGF antibodies vs none) before randomisation. For on-site screening participants, we are using a second randomisation schedule to assign them (1:1 ratio) to OCT or no OCT. Block randomisation in groups of four will be done: each block will include two usual screening assignments, one NM UWF assignment and one NM UWF+OCT assignment. To ensure that randomisation is concealed, all study personnel other than the biostatistician (study coordinator, staff who perform NM UWF imaging and OCT and the study ophthalmologist) and personnel involved in participants' diabetes care (endocrinologists, nurses, dieticians, family physicians and non-study eye care professionals) do not have access to the randomisation schedules.

\section{Interventions}

The participant timeline and data collection protocol are available in the online supplementary file (online supplementary appendices 2 and 3).

\section{Usual screening group}

We have designed the usual screening strategy to mimic the current practice in Ontario. Participants due for eye screening according to the CDA guidelines are encouraged by their endocrinologist at their diabetes clinic visit to arrange an exam with an eye care professional. In Ontario, screening exams are paid for by the government and most are done by optometrists. We are explaining the reason for the exam - to detect clinically silent disease for which treatments to protect vision may be needed-and are providing contact information for London optometrists to participants who do not have an identified eye care professional. When seen for screening, the specific screening method performed is at the eye care professional's discretion but usually includes a form of dilated retinal examination. If clinically important findings are noted, further follow-up and/or management is arranged depending on severity and ranges from a repeat eye exam at a sooner interval to referral to ophthalmology for treatment. At all follow-up diabetes clinic visits within a year of randomisation, participants' status with respect to eye screening is reviewed and, if not done, participants are being encouraged to arrange an eye exam.

\section{On-site screening group}

Participants randomised to on-site screening are immediately undergoing NM UWF imaging in the ophthalmology department within the same hospital. Half of participants in the on-site screening group are also undergoing OCT, but because we lack same-day access to OCT and because assessment of the added value of OCT to NM UWF imaging is a secondary objective, the OCTs are being batched for completion within 2 weeks of randomisation. To better isolate any incremental effect of NM UWF imaging to detect eye disease over current screening, we are encouraging participants randomised to on-site screening to also undergo eye screening by the CDA guidelines. Thus, the on-site screening participants are being advised to arrange an exam with an eye care professional as described above for usual screening participants.

\section{Outcomes}

The primary outcome is AED as defined by diabetic eye disease for which assessment by an ophthalmologist and/or increased ocular surveillance is indicated. For all participants, the primary outcome can occur at any time between randomisation and 1year later, but the definition of AED differs by group.

For usual screening participants, AED is based on the exam findings and recommendations about patient disposition (ie, management and/or follow-up required) as determined by a non-study eye care professional. Documentation of a screening exam by an eye care professional requires that findings and recommendations be provided in a written report where the participants are asked for the name of their usual eye care professional and the study team will contact them to request the report. Any of the following are considered indicative of AED if included on the report: moderate or severe non-proliferative DR, any proliferative DR, CSME, referral to an ophthalmologist and re-examination in $<12$ months. For on-site screening participants, AED is based on interpretation of NM UWF images by the study ophthalmologist and requires at least one finding listed in table 1.

The prespecified secondary outcomes are:

A. Screening adherence as determined by: (i) the proportions of participants who have screening completed within 1 year of randomisation by the primary screening method, viz., NM UWF images for the on-site screening group or an eye exam by

\begin{tabular}{ll}
\hline $\begin{array}{l}\text { Table } 1 \\
\text { participants }\end{array}$ Undergoing on-site screening \\
\hline $\begin{array}{l}\text { Diabetic } \\
\text { retinopathy }\end{array}$ & $\begin{array}{l}\text { Intra-retinal microvascular abnormalities } \geq 2 \\
\text { quadrants } \\
\text { Venous beading } \geq 2 \text { quadrants }\end{array}$ \\
& $\begin{array}{l}\text { Neovascularisation elsewhere or } \\
\text { neovascularisation of the disc }\end{array}$ \\
& $\begin{array}{l}\text { Retinal haemorrhage in all four quadrants } \\
\text { Vitreous haemorrhage }\end{array}$ \\
Diabetic & $\begin{array}{l}\text { Microaneurysms, retinal haemorrhages or } \\
\text { maculopathy } \\
\text { exudes within one disc diameter of fovea } \\
\text { (ie, centre-involved macular oedema or } \\
\text { clinically significant macular oedema, as per } \\
\text { the Early Treatment Diabetic Retinopathy } \\
\text { Study classification) }\end{array}$ \\
\hline
\end{tabular}

AED is based on interpretation of NM UWF images by a retina specialist and requires that at least one finding be present. 
an non-study eye care professional for the usual screening group; (ii) the proportion of participants in the on-site screening group who have also had a screening eye exam by a non-study eye care professional within 1 year of randomisation. For (i) and (ii) above, documentation of a screening exam by an eye care professional requires that the exam findings and follow-up recommendations be provided by the eye care professional in a written report.

B. DME as defined by: (i) the proportions of participants with CSME detected by the primary screening method, viz., NM UWF images (on-site screening group) or an eye examination by a nonstudy eye care professional (usual screening group); (ii) the proportions of participants with DME detected by NM UWF imaging alone versus NM UWF imaging plus OCT; and (iii) the proportions of participants with DME detected by NM UWF imaging plus OCT versus an eye exam by a nonstudy eye care professional. The definition of DME by OCT requires the presence of one or more of an intraretinal cyst, intraretinal exudate or subretinal fluid.

\section{Strategies to limit bias}

Certain biases are possible because masking participants, the study coordinator and the outcome assessors to the screening groups is not feasible.

One source of potential bias is unequal use between the two groups of treatments that affect progression of diabetic eye disease. These cointerventions include treatments to control blood glucose and blood pressure and to promote smoking cessation and fenofibrate. To limit this, all personnel involved in participants' diabetes care are not being informed about participants' screening group assignments. The study coordinator is also encouraging participants to not disclose this information to their caregivers. In addition, we are recording the relevant cointerventions and their effects (A1C and blood pressure levels; smoking status) among participants from baseline through 1 year and will use this information to adjust analyses that compare AED rates between on-site and usual screening groups.

The potential for unequal surveillance for diabetic eye disease between the two screening groups because of more frequent ophthalmoscopy by participants' endocrinologists and primary care providers is unlikely. In Ontario, dilated pupil fundoscopic exams are not normally done when patients with diabetes attend endocrinology and primary care outpatient clinics. Exceptions occur (eg, patients with new visual complaints), but even then, the exam is usually by direct ophthalmoscopy through undilated pupils, is insensitive in detecting diabetic eye disease and does not forego a recommendation that the patient still see an eye care professional. To assess for possible surveillance bias, we are recording whether eye exams have been
Table 2 The Diabetic Retinopathy Disease Severity Scale

Findings observable on dilated

Disease severity level ophthalmoscopy

\begin{tabular}{|c|c|}
\hline $\begin{array}{l}\text { No apparent } \\
\text { retinopathy }\end{array}$ & No abnormalities \\
\hline $\begin{array}{l}\text { Mild non-proliferative } \\
\text { DR }\end{array}$ & Microaneurysms only \\
\hline $\begin{array}{l}\text { Moderate non- } \\
\text { proliferative DR }\end{array}$ & $\begin{array}{l}\text { More than just microaneurysms but } \\
\text { less than severe non-proliferative } \\
\text { DR }\end{array}$ \\
\hline $\begin{array}{l}\text { Severe non- } \\
\text { proliferative DR }\end{array}$ & $\begin{array}{l}\text { Any of the following and no signs of } \\
\text { proliferative retinopathy: } \\
\text { - } 20 \text { intraretinal haemorrhages and } \\
\text { microaneurysms in each of the four } \\
\text { quadrants } \\
\text { - Definite venous beading in } \geq 2 \\
\text { quadrants } \\
\text { - Prominent intraretinal } \\
\text { microvascular abnormalities in } \geq 1 \\
\text { quadrant }\end{array}$ \\
\hline Proliferative DR & $\begin{array}{l}\text { One or more of the following: } \\
\text { - Neovascularisation } \\
\text { - Vitreous/preretinal haemorrhage }\end{array}$ \\
\hline
\end{tabular}

DR, diabetic retinopathy.

done by participants' endocrinologists and primary care providers during follow-up.

With respect to biased assessment of the primary outcome of AED between screening groups, for usual screening participants, AED is defined by findings by non-study eye care professionals who are not being made aware of the study. In addition, criteria to define AED among usual screening participants are based on objective findings that are included in a standardised clinical classification scale in wide use (the Diabetic Retinopathy Disease Severity Scale (DRDSS)) (table 2) ${ }^{23}$ In our pilot study, we found that optometrists in our region usually reported their exam on a template similar to DRDSS.

For on-site screening participants, AED is based on NM UWF images as read by a retina specialist with experience with NM UWF imaging. Similar to usual screening participants, the criteria to diagnose AED by NM UWF images are objective and are also generally consistent with DRDSS.

\section{Sample size}

Detailed sample size considerations that include our pilot study findings are given in online supplementary appendix 4. We are randomising 740 participants to obtain $80 \%$ power to identify at least a $5 \%$ absolute increase in rate of detection of AED among on-site screening versus usual screening participants. This difference translates into a number-needed-to-screen by on-site screening of 20 to detect 1 additional person with AED versus usual screening and corresponds to a relative increase in detection of AED by NM UWF imaging of $60 \%$. We judge this 'effect size' to be clinically important and plausible in absolute and relative terms. 


\section{Statistical analysis}

The statistical analysis plan is detailed in online supplementary appendix 5. The primary analysis will use an intention-to-screen approach, viz., attribution of AED will be to the screening group (on-site or usual) to which participants were randomised. Data from all randomised participants will be included in the primary analysis other than participants who are found, after randomisation, to have undergone a screening exam by an eye care professional within 12 months of entry. The primary analysis will assess the unadjusted proportions of AED between on-site and usual screening groups by Pearson's $\chi^{2}$ or Fisher's exact test as appropriate and will test a superiority hypothesis in favour of on-site screening at a two-tailed significance level of $5 \%$.

For participants with missing primary outcome data, sensitivity analyses comparing the proportions of AED between on-site and usual screening groups with imputation of missing outcomes by "worst-case/best-care scenarios' will be done. The impact of baseline imbalances on AED rates between on-site and usual screening groups will be assessed in a logistic regression analysis that adjusts for baseline A1C level, previous treatment for diabetic eye disease, blood pressure level, smoking status, fenofibrate treatment, age and gender.

\section{Ethics and dissemination of results}

The pilot study and Clearsight trial protocols have been approved by the Western University research ethics board. All participants in the pilot study provided informed consent, and provision of informed consent is an entry criterion for the Clearsight trial. A sample letter of information and consent form is available in online supplementary appendix 6. The findings of the Clearsight trial will be disseminated directly to participants and through peer-reviewed publications and conference presentations.

We have not constituted a data monitoring committee as we will not perform interim analyses for the purpose of stopping or extending the trial and there are no known risks from NM UWF imaging and OCT. Both the usual screening and on-site screening groups are receiving standard-of-care advice about the need to be screened by an eye care professional. For on-site screening participants in whom AED is seen on UWF imaging or in whom DME is seen on OCT, a letter is being sent to the participant's eye care professional informing them of the findings.

\section{Pilot study}

The pilot study aims were to gain experience with NM UWF imaging; assess patients' understanding of the need for eye screening, perceived screening barriers and acceptance of NM UWF imaging using self-completed questionnaires; assess patients' adherence to screening based on the self-reported date of their last eye exam and by directly contacting their eye care professional; and obtain information needed to estimate the sample size for the Clearsight trial including the rate for the primary outcome (AED) in control participants. We also assessed the intraobserver agreement for NM UWF to detect AED by NM UWF imaging.

The pilot study involved 135 patients with diabetes who underwent NM UWF imaging by the Optos 200Tx retinal camera ${ }^{20}$ immediately after their diabetes clinic appointment. We also reviewed 229 consecutive charts of additional patients with diabetes who had dilated pupil screening exams in a local optometry office. To assess the intraobserver agreement for AED by NM UWF imaging, we determined the phi statistic for the Clearsight study ophthalmologist who read and reread 134 randomly sorted NM UWF pilot study images. ${ }^{24}$

Of 135 pilot study patients undergoing NM UWF imaging (74 (55\%) male and $68(50 \%)$ with T1D; mean (SD) age 53.1 (16.2) years, diabetes duration 20.6 (11.6) years and A1c $7.9 \%$ (0.012)), gradable images were obtained in both eyes in 134, and 135 completed the questionnaire. A readable NM UWF image was obtained in over $90 \%$ of patients within $30 \mathrm{~min}$ of being offered the test; $99 \%$ agreed/strongly agreed that 'having a yearly eye exam is important', $16 \%$ agreed/strongly agreed that they 'often have difficulty scheduling yearly eye exams due to time constraints', 96\% agreed/strongly agreed that 'having my eyes photographed on the same day as my diabetes clinic visit was convenient' and $82 \%$ agreed/ strongly agreed with the statement 'I would prefer to have eye screening using this camera over a standard eye exam by an eye-care professional'. The mean (SD) time to their last eye exam was 10 (8.7) months (range 0-46 months), but $44 / 135(33 \%)$ patients indicated they had not seen an eye care professional for over a year.

Out of 134 patients, $13 \quad(9.7 \% ; 95 \%$ confidence limits (CLs) $5.5 \%, 16.3 \%$ ) were found to have AED by NM UWF imaging as it is being defined for on-site screening participants in Clearsight. In the chart review of 229 patients with diabetes having screening eye exams in a London optometry office, $3.9 \% \quad(95 \%$ CLs $1.9 \%, 7.6 \%$ ) were found to have AED based on the optometrist's report as it is being defined in usual screening participants in Clearsight. The intraobserver reliability by the Clearsight study ophthalmologist to identify AED versus no AED based on reading $134 \mathrm{NM}$ UWF images was substantial (phi statistic=0.67).

\section{DISCUSSION}

Overcoming the lost opportunity to prevent vision loss in people with diabetes because of suboptimal eye screening is a major challenge. ${ }^{2} 68$ Many factors contribute to the gap in care between identifying asymptomatic at-risk patients through screening and providing vision-saving treatments to those at risk. While screening strategies currently recommended by major practice guideline groups have in common visualisation of the retina every 1-2 years, substantial differences exist about how to do this. The uncertainty reflects in part a tradeoff between simpler methods that reduce patient and healthcare system barriers to screening and 
more intensive, often costlier methods that yield better discriminative accuracy (ie, the capacity to distinguish patients with eye disease needing closer follow-up and treatment from those who do not). The uncertainty may also reflect the need for high-quality evidence including pragmatic randomised trials ${ }^{25}$ comparing different screening strategies under conditions that approximate 'real life', and we have designed Clearsight with this need in mind.

Studies using patient questionnaires, focus group discussions, structured interviews and randomisation have shown that mydriasis, the extra time patients need to attend eye screening exams, and the failure to integrate screening into routine diabetes care visits negatively affect adherence. ${ }^{9-15}$ We chose to study NM UWF imaging based on its potential to overcome these barriers without clinically important losses in discriminative accuracy. The absence of mydriasis is an obvious advantage, but we also did not find it difficult in our pilot study to incorporate 'on-demand' NM UWF imaging into a busy diabetes outpatient clinic in patients who, at the time of their appointment, were due for eye screening. In our pilot study, we obtained interpretable NM UWF images in over $90 \%$ of patients within $30 \mathrm{~min}$ of it being offered and also found that the patients were pleased with the convenience this afforded.

Regarding NM UWF imaging accuracy relative to other screening tests, the current evidence is significant in both amount and quality. The evidence is also reassuring in ruling out major deficiencies in NM UWF imaging's capacity to detect DR compared with accepted practice (dilated pupil exams by experienced ophthalmologists; dilated pupil digital retinal photographs) and reference (dilated pupil 7-field fundus photography) standard screening tests. ${ }^{16-19}$ In fact, one can argue that the evidence has reached a point where NM UWF imaging should now be recommended over other screening tests for DR and that a randomised trial comparing NM UWF imaging to other tests is not needed. For example, in the studies comparing NM UWF images to dilated pupil 7-field fundus photographs, the levels of agreement to detect and grade DR were substantial (kappa values $\geq 0.77^{18}{ }^{19}$ ) and the sensitivity $(99 \%)$ and specificity $(100 \%)$ for no DR versus any DR were particularly high. ${ }^{19}$ On the other hand, we found the evidence to be less certain in respect to NM UWF imaging's capacity to detect clinically important DME. ${ }^{16-19}$ We note also that British, American and Canadian practice guideline groups have not included NM UWF imaging as a screening option ${ }^{3-5}$ despite the compelling, but observational, evidence and that a randomised trial that confirms (or refutes) whether NM UWF imaging is superior to other screening tests will yield stronger information on the question.

To our knowledge, there has been no randomised trial comparing NM UWF imaging to the gold standard of 7-field fundus photography, and one could argue that this comparison would be the ideal one to evaluate.
However, most people with diabetes do not have routine 7-field fundus photography for retinopathy screening, and as noted above, there is good evidence to validate the accuracy of NM UWF imaging against 7-field fundus photography.

Limitations to the Clearsight trial include the potential for bias because of differences in how we are ascertaining AED between on-site and usual screening groups, plus the fact that we cannot fully mask outcome assessors to screening group assignment. We have taken steps in the trial's design to reduce this possibility. As a second limitation, Clearsight is neither large nor long enough to test whether NM UWF imaging improves visual outcomes. Should we find that NM UWF imaging improves AED detection compared with usual screening, the inference that it also improves outcomes through earlier use of effective therapy will, though plausible, be unproven. Third, Clearsight has not been designed to determine which components of on-site screening (the NM UWF imaging procedure, the convenience of eye screening on the same day as the diabetes visit or both) affect screening adherence. Fourth, generalising results of Clearsight beyond the diabetes referral outpatient setting in which it is being undertaken to primary care settings, where most eye screening occurs, will be subject to qualification. Finally, the results of Clearsight may not be generalisable to places (eg, UK) where photographic retinopathy screening is the standard of care.

In conclusion, NM UWF imaging is a highly promising screening option for diabetic eye disease. The Clearsight trial will determine whether NM UWF imaging improves detection of clinically important eye disease compared with current practice and, if so, should help address an important gap in diabetes care.

Acknowledgements For the pilot study, we thank Devin Fisher and Richard Filek for participant recruitment and NM UWF image procurement, Dr Cheryl Letheren and associates for review of their optometry charts and Leslie Pal and Sue Tereschyn for study coordination. Acknowledgement is also given to the Schulich School of Medicine \& Dentistry for research fellowship support to SLL.

Contributors JLM and SLL conceived the study hypothesis. SLL, LWM, IMH, JRG and JLM contributed to the design and execution of the pilot study. SLL wrote the first draft of the trial protocol, and all authors provided critical revision of the protocol. JLM wrote the first draft of the manuscript. SLL, LWM, NSK, DSC, JRG and IMH reviewed and edited the manuscript. All authors approved the final manuscript.

Funding The funding sources for both the pilot study and the Clearsight trial had no role in the design of the study and will not have any role in the performance of the study.

Competing interests None declared.

Ethics approval Western University health science research ethics board.

Provenance and peer review Not commissioned; externally peer reviewed.

Open Access This is an Open Access article distributed in accordance with the Creative Commons Attribution Non Commercial (CC BY-NC 4.0) license, which permits others to distribute, remix, adapt, build upon this work non-commercially, and license their derivative works on different terms, provided the original work is properly cited and the use is non-commercial. See: http://creativecommons.org/ licenses/by-nc/4.0/

(c) Article author(s) (or their employer(s) unless otherwise stated in the text of the article) 2017. All rights reserved. No commercial use is permitted unless otherwise expressly granted. 


\section{REFERENCES}

1. Klein R, Klein BE. Diabetic eye disease. Lancet 1997;350:197-204.

2. Cruess AF, Gordon KD, Bellan L, et al. The cost of vision loss in Canada. 2. results. Can J Ophthalmol 2011;46:315-8.

3. Boyd SR, Advani A, Altomare F, et al. Canadian Diabetes Association 2013 clinical practice guidelines for the prevention and management of diabetes in Canada: retinopathy. Can J Diabetes 2013;37(suppl 3):S332-S333.

4. National Institute for Health and Care Excellence (NICE). Type 2 diabetes in adults: management (NICE Guideline 28). 2015 https:// www.nice.org.uk/guidance/ng28.

5. The American Diabetes Association. Standards of medical care in diabetes. Diabetes Care 2016;39(suppl 1):S1-S119.

6. Booth GL, Polsky JY, Dozdyra G, et al. Regional measures of diabetes burden in Ontario. Toronto, ON: Institute for Clinical Evaluative Sciences, 2012.

7. Kiran T, Kopp A, Moineddin R, et al. Unintended consequences of delisting routine eye exams on retinopathy screening for people with diabetes in Ontario, Canada. CMAJ 2013;185:E167-E173.

8. Scanlon PH, Stratton IM, Leese GP, et al. Screening attendance, age group and diabetic retinopathy level at first screen. Diabet Med 2016;33:904-11.

9. Murgatroyd H, MacEwen C, Leese GP. Patients' attitudes towards mydriasis for diabetic eye disease screening. Scott Med J 2006;51:35-7.

10. Dervan E, Lillis D, Flynn L, et al. Factors that influence the patient uptake of diabetic retinopathy screening. Ir J Med Sci 2008;177:303-8.

11. Lewis $\mathrm{K}$, Patel D, Yorston $\mathrm{D}$, et al. A qualitative study in the United Kingdom of factors influencing attendance by patients with diabetes at ophthalmic outpatient clinics. Ophthalmic Epidemiol 2007; $14: 375-80$.

12. Silva PS, Cavallerano JD, Aiello LM, et al. Telemedicine and diabetic retinopathy: moving beyond retinal screening. Arch Ophthalmol 2011;129:236-42.

13. Wilson $\mathrm{C}$, Horton M, Cavallerano J, et al. Addition of primary carebased retinal imaging technology to an existing eye care professional referral program increased the rate of surveillance and treatment of diabetic retinopathy. Diabetes Care 2005;28:318-22.
14. Mansberger SL, Gleitsmann K, Gardiner S, et al. Comparing the effectiveness of telemedicine and traditional surveillance in providing diabetic retinopathy screening examinations: a randomized controlled trial. Telemed J E Health 2013;19:942-8.

15. Conlin PR, Fisch BM, Cavallerano AA, et al. Nonmydriatic teleretinal imaging improves adherence to annual eye examinations in patients with diabetes. J Rehabil Res Dev 2006;43:733-40.

16. Neubauer AS, Kernt M, Haritoglou C, et al. Nonmydriatic screening for diabetic retinopathy by ultra-widefield scanning laser ophthalmoscopy (Optomap). Graefes Arch Clin Exp Ophthalmol 2008;246:229-35.

17. Wilson PJ, Ellis JD, MacEwen CJ, et al. Screening for diabetic retinopathy: a comparative trial of photography and scanning laser ophthalmoscopy. Ophthalmologica 2010;224:251-7.

18. Kernt M, Hadi I, Pinter F, et al. Assessment of diabetic retinopathy using nonmydriatic ultra-widefield scanning laser ophthalmoscopy (Optomap) compared with ETDRS 7-field stereo photography. Diabetes Care 2012;35:2459-63.

19. Silva PS, Cavallerano JD, Sun JK, et al. Nonmydriatic ultrawide field retinal imaging compared with dilated standard 7-field $35-\mathrm{mm}$ photography and retinal specialist examination for evaluation of diabetic retinopathy. Am J Ophthalmol 2012;154:549-59.

20. Optos Ultra-Widefield Imaging. http://www.optos.com/en-CA/retinalimaging-products2/. (accessed Nov 2016).

21. Klein R, Klein BE, Moss SE, et al. The Wisconsin Epidemiologic Study of Diabetic Retinopathy. XV. The long-term incidence of macular edema. Ophthalmology 1995;102:7-16.

22. Virgili G, Menchini F, Murro V, et al. Optical coherence tomography (OCT) for detection of macular oedema in patients with diabetic retinopathy. Cochrane Database Syst Rev 2011:CD008081.

23. Wilkinson CP, Ferris FL, Klein RE, et al. Proposed international clinical diabetic retinopathy and diabetic macular edema disease severity scales. Ophthalmology 2003;110:1677-82.

24. Guyatt G, Rennie E, Meade MO, eds. Users' Guide to the Medical Literature: A Manual for Evidence-Based Clinical Practice. 2nd ed. New York, NY: McGraw-Hill, 2008.

25. Patsopoulos NA. A pragmatic view on pragmatic trials. Dialogues Clin Neurosci 2011:13:217-24. 